\title{
Dynamic Prediction of Energy Delivery Capacity of Power Networks: Unlocking the Value of Real-Time Measurements
}

\author{
Authors \\ Peter Schell Ampacimon, Lawrence Jones Alstom Grid , Philippe Mack Pepite, \\ Bertrand Godard and Jean-Louis Lilien, University of Liège
}

\section{Introduction}

Today and tomorrow even more, each day is different ${ }^{1}$ and this calls for a change in the way our electricity networks (both transmission and distribution) must be operated to continue to guarantee the security of electric energy supply that our economy depends on.

Furthermore, in many parts of the world this change requires that transfer capacities of electricity networks be extended dramatically and very quickly if we are to meet the sustainability objectives as set forward by the different governments of the world (e.g. the 20/20/20 objectives of the European Union, see for example [4].

One "solution" that has been put forward is to increase the network's system capacity by making it "smarter", i.e. by gathering more information about the system and the assets to allow the operators to make better decisions and in this sense increase the efficiency of existing assets. This is complementary to the "classic" approach of increasing capacity by investing in new assets, which in many parts of the world is becoming increasingly a difficult and slow process. While, measuring data on the behavior of the grid is good, it will not be of much use if grid operators are unable to use this data to predict the trajectory of the system states and therefore help the grid operators make better informed real-time decision. TSO more accurate predictions system operation is a ultimate benefit of deploying the state-of-the-art analytical tools in smarter electric grids. The predicted capacity can be integrated with the Energy Management System and other decision support tools used in control centers to operate the power grid.

This paper describes recent advances in prediction software using advanced data-mining tools, and shows how this can significantly increase the operational value of the data gathered. These new tools allow for new operational procedures to be defined that increase the usages of the network without decreasing security of supply. We will use the example of dynamic line rating, i.e. determining the thermal rating of an overhead line based on real-time environmental parameters (temperature, wind speed \& direction and solar radiation) to highlight how the use of numerical data-mining tools can help us reliably predict future behavior of power networks.

\section{Context}

The results described in this paper are based on measurements taken from a set of $18 \mathrm{~km}$ long $150 \mathrm{kV}$ overhead lines between Brugge and Slijkens (Figure 1) near the Belgian coast and a $225 \mathrm{kV}$ much longer line in Britany (Figure 2), France to the north-west of Nantes also near the coast.

\footnotetext{
${ }^{1}$ Uncertainties are not only affecting load/injection but also actual properties (like capacity) of equipment/assets. These can vary with time (ageing) or with environmental conditions. Being able to understand and predict these uncertainties is a cost effective way to increase asset efficiency and leverage the non-working capital imposed by current higher security margins
} 


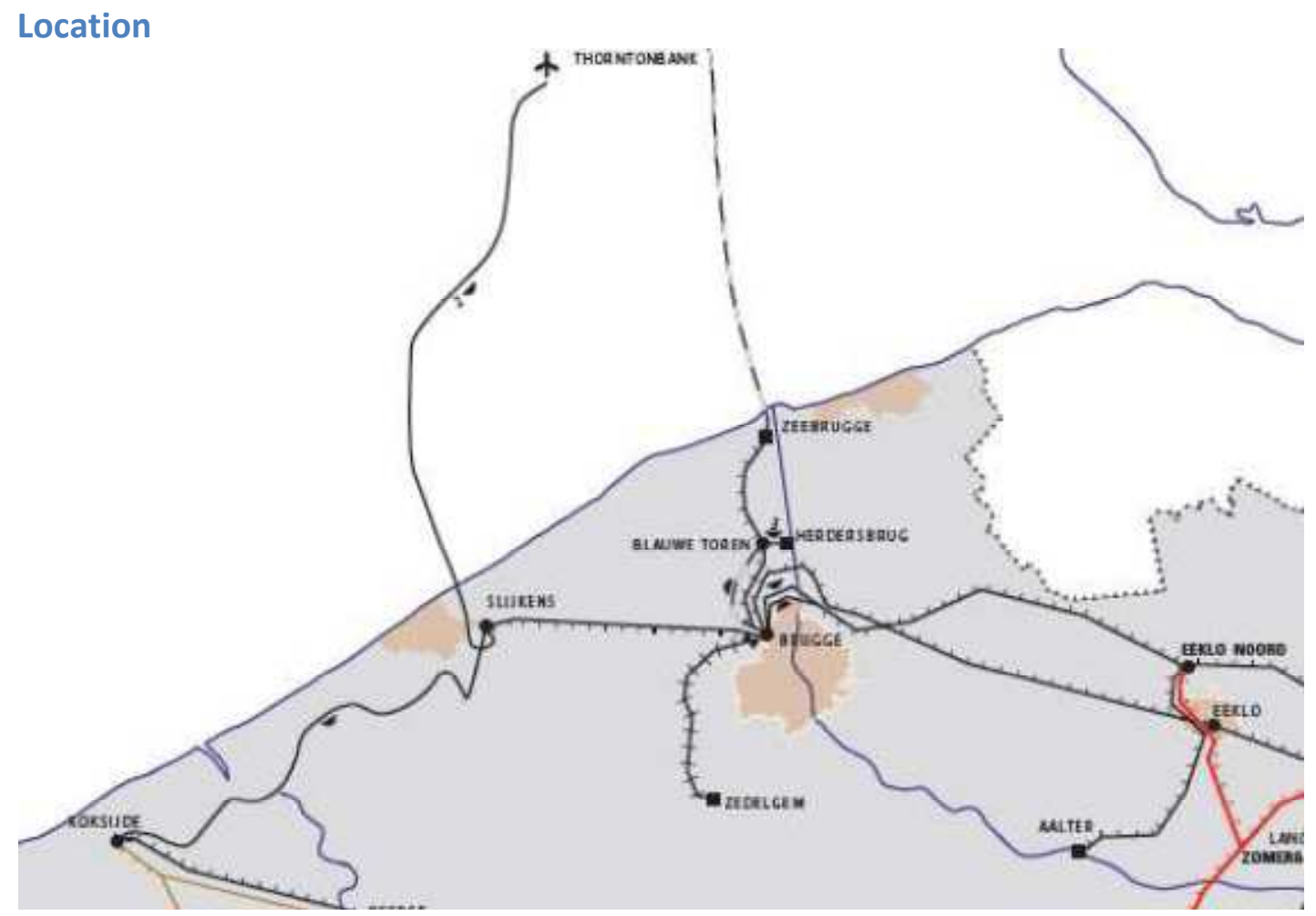

Figure 1 : Elia Brugge-Slijkens Line in Belgium. Wind farm can be seen in the North sea ("Thornton bank"). The Belgium coast is visible on the figure with a small part of Belgium network showing some $150 \mathrm{kV}$ lines (in black) and some $400 \mathrm{kV}$ lines (in red). The Brugge Slijkens $150 \mathrm{kV}$ lines equipped with sensors are clearly visible.

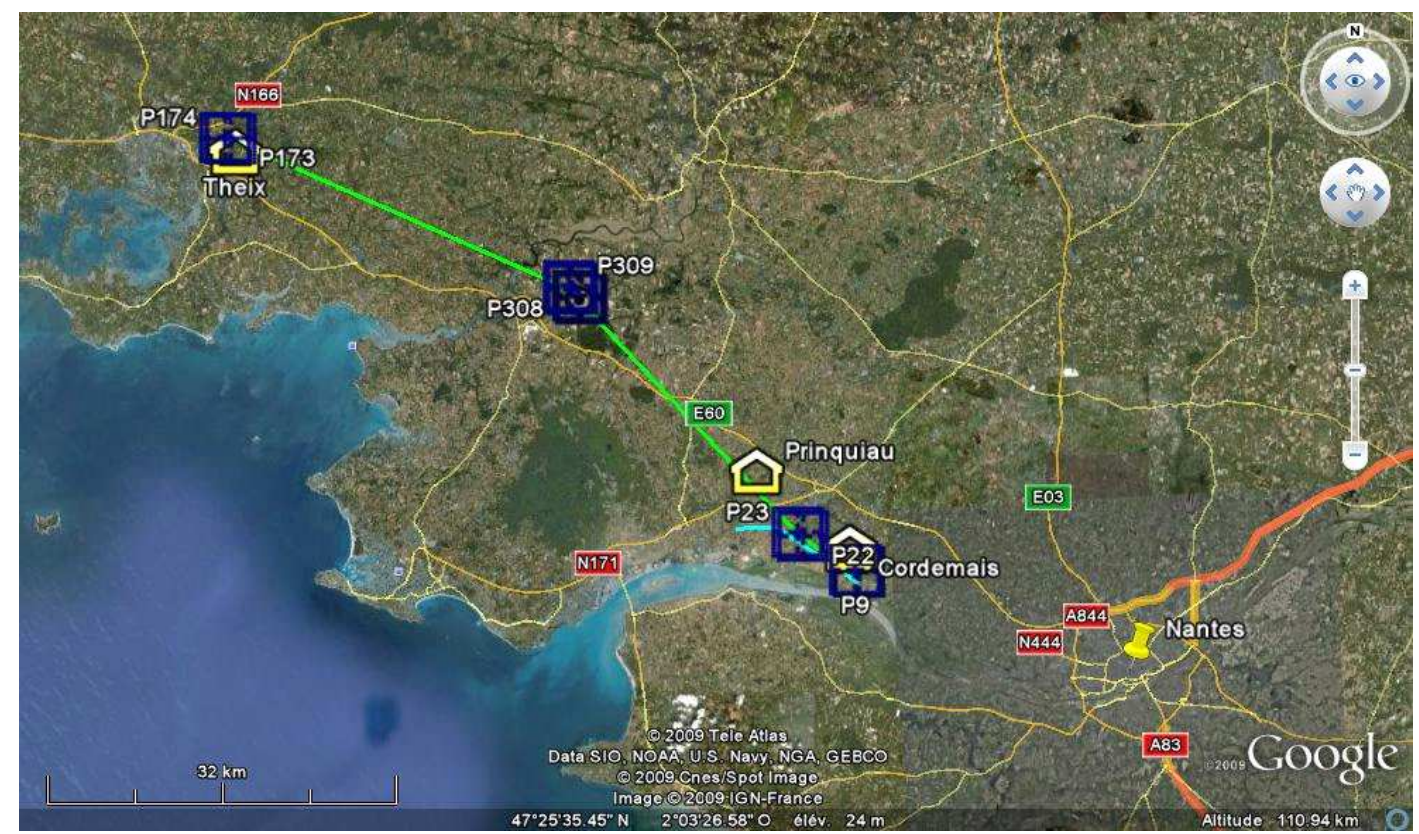

Figure 2: The equipped (with sensors) $225 \mathrm{kV}$ line of RTE between Nantes (Cordemais) and Theix in Brittany, France. This is a google earth view to be able to locate the relative distance between Atlantic Ocean and the line highlighted by the the green straight lines.

\section{Business needs for DLR Sensors}


The business need that has driven the installation of Dynamic Line Rating sensors on these lines is the integration of large renewable (wind) energy sources. The aim is to find a solution to the challenges posed by this integration, namely the rapid pace at which increased network system capacity is required, the need to find economically optimal solution to the intermittent loads and keep investment reasonable and of course the need to guarantee security of supply at all times. Dynamic Line Rating, i.e. determining the thermal rating of a line based on actual measurements instead of the classic annual or seasonal static rating, offers one solution that meets the challenges above. One major issue that remains is to allow the grid operators to fully take advantage of this relatively inexpensive (compared to alternatives) source of extra network capacity, and improve operational efficiency of other grid assets is namely the capability to accurately and reliably predict the dynamic behavior of the thermal rating for the coming hours and dayahead.

Being able to predict future situations is a challenge faced by TSOs today. Predicting physical properties of the grid, like ampacity, is key to operate the system closer to the actual limits. In the past, the load patterns were the main source of critical uncertainties for the security of the system. Nowadays critical uncertainties are numerous due to fast changing market conditions and increasing weight of renewable energy. To predict the behavior of the system it is key to take into account the main drivers of uncertainties like weather forecast, market conditions and customer behavior (demand side management). The methods described in this paper could be transposed in other problems where reliable prediction is strongly impacted by weather conditions, like renewable energy production.

\section{Tools used}

\section{Acquire Data with DLR}

A direct Dynamic Line Rating (DLR) system as used in this case [1,2] continuously measures sag of all critical spans. The chosen sensor determines real time sag data directly out of the vibration frequency spectrum of the span (more details on [6] ). This unique and original physical approach allows one to monitor the sag of critical spans without any parameters set. So no calibration is needed. Therefore the system is absolutely reliable while being very simple to install and operate.
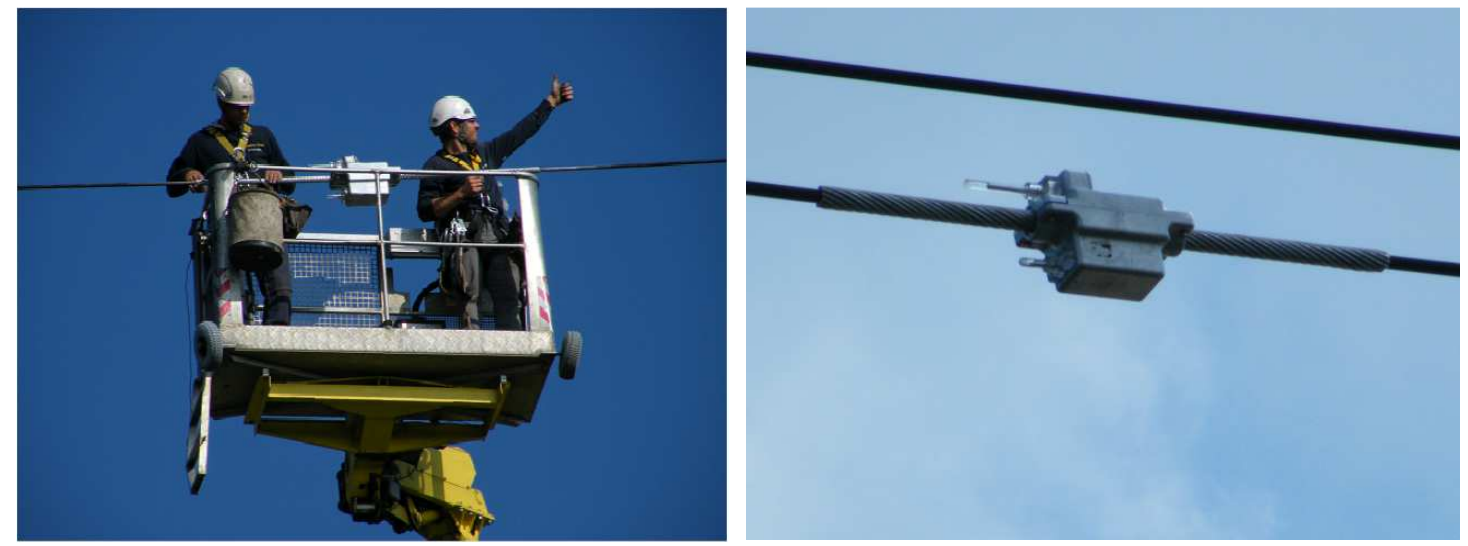

Figure 3 : (left) Linesman in the elevator notifying another linesman in the mast that everything is ok during installation and (right) the sensor as installed on the line with armor rods.

The Real Time (RT) current carrying capacity also called Ampacity (Ampere Capacity) of each potentially critical span $^{2}$ is determined for the maximum sag or the maximum conductor temperature whichever comes first. The Ampacity is determined using international recommendations by CIGRE and IEEE with effective weather data (wind speed and solar radiation) based on the sag observation. The RT Line Ampacity is naturally the minimum Ampacity of all the monitored/observed spans.

\footnotetext{
${ }^{2}$ Critical spans will represent the spans for which, if the clearances are respected, the clearances will be respected on the rest of the line. Determining which is (are) the critical span(s) needs to be done separately for each line.
} 
The basic output of the system are real-time ampacity (also called real time thermal rating) and sag. Historical records and statistics are used to evaluate the long-term thermal rating potential and feed prediction algorithms in order to perform short and medium term (a few minutes to a few hours) predictions of ampacity. It is also possible to combine these historical records with weather forecast and use the latest machine learning algorithms to perform long term (up to 2 days) ampacity forecast as described in this paper.

\section{Short-term forecast ( $1 \mathrm{~h} / 4 \mathrm{~h})$ of network capacity}

A specific new predictive model for short term Ampacity prediction was developed (up to 4 hours) based on Real Time Sag measurement data. This model is based on a combination of (i) historical records of real time ampacity and (ii) historical records of effective ${ }^{3}$ weather data ; both obtained by real time sag observations.

Similar period to actual situation in the recent historical behavior of the line are used/combined to calculate ampacity prediction ( $k$-nearest neighbors algorithm) and statistics on historical records of effective weather data can be used to determine maximal deterioration in the onsite weather conditions. This model is based on the fact that weather conditions have some "persistence" and that power lines sag evolves with a rather long time constant (typically 15 minutes, which means that new equilibrium position after drastic transient of either current load or weather conditions would need around 4 times that constant before being established, or near 1 hour). Moreover local effects (line screening effects by trees, local wind speed and/or direction effects, etc) are obviously hidden in the past behavior of the line for similar events and such analysis which needs to be done for each critical span may lead to much longer time prevision with a very good estimate. (4 hours as indeed observed in practical cases).

In consequence, short and medium term prediction can be calculated with no need for weather forecast data. This of course needs appropriate data mining analysis and is very much dependent of a specific installation on which some weeks up to some months of observations are needed.

Figure 4 shows a real 7 days period comparison between the real time ampacity and the one-hour and four hours forecasted ampacity by the developed algorithm (This is on the $150 \mathrm{kV}$ line, Belgium, ELIA used as Demo for European TWENTIES project [4]). These results clearly show a good consistency between ampacity and forecasted ampacity.

\footnotetext{
${ }^{3}$ With effective we mean as sensed by the measured span as a whole. The weather parameters are "recalibrated" to meat the measured sag for a given current and represent the correct mean values (in time \& space) for the instrumented span
} 


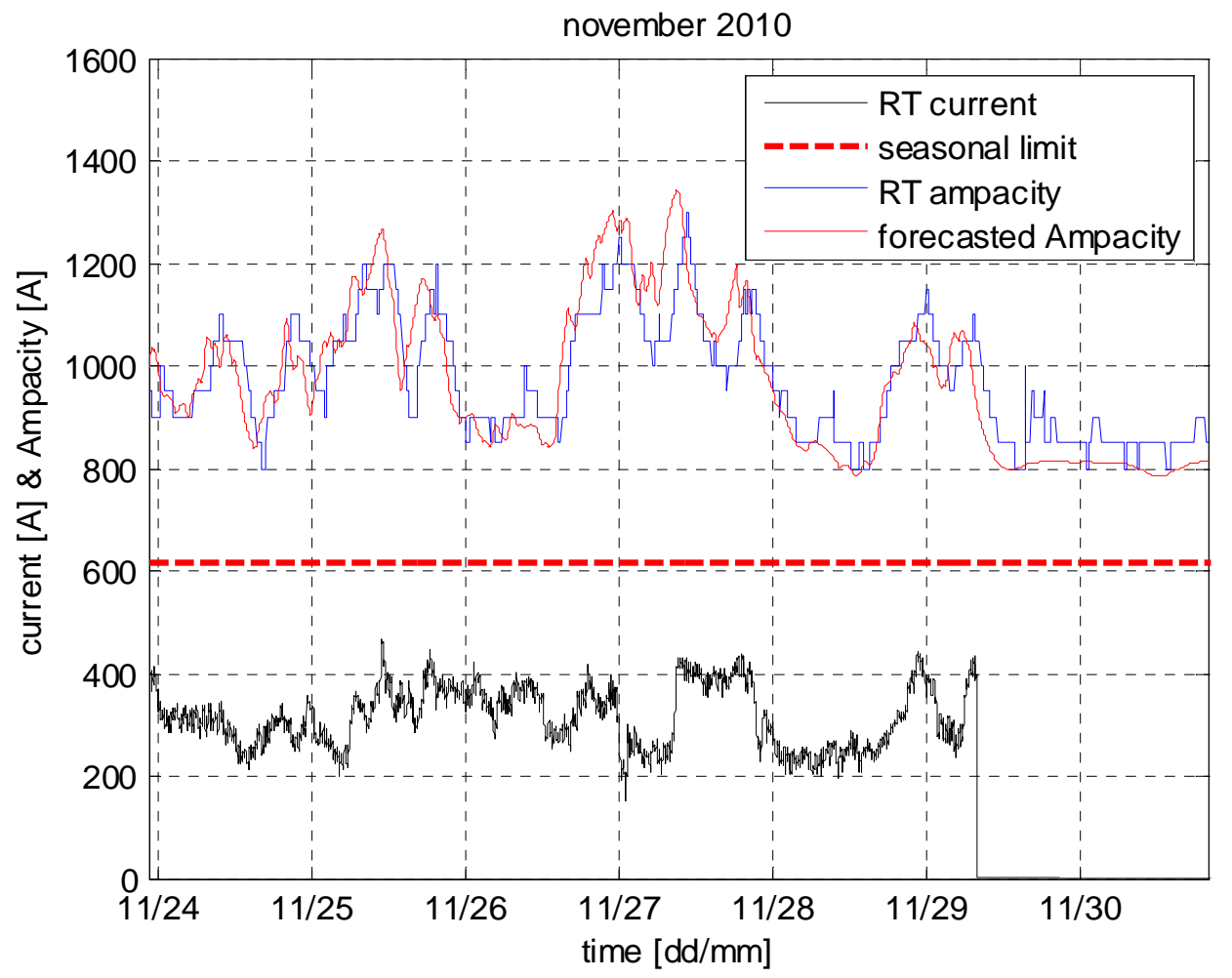

Figure 4 : Current, seasonal limit, real time Ampacity, and superimposed short term (4 hours) forecasted ampacity by Ampacimon algorithm.

\begin{tabular}{|c|c|c|c|c|c|c|c|c|}
\hline & \multicolumn{8}{|c|}{ error bins [A] (error = actual rating - forecasted ampacity) } \\
\hline & \multicolumn{8}{|c|}{ Overestimated ( error $<0$ ) / underestimated ( error $>0$ ) } \\
\hline & ]$-125,-75]$ & ]-75,-25] & ]$-25,25[$ & {$[25,75[$} & {$[75,125[$} & {$[125,175[$} & {$[175,225[$} & {$[225,275[$} \\
\hline $\begin{array}{l}\% \text { of } \\
\text { occurrence }\end{array}$ & 2 & 11 & 35 & 25 & 12 & 8 & 5 & 2 \\
\hline
\end{tabular}

Table 1 : statistics of error between forecasted ampacity and actual rating.

Table 1 shows statistics of the difference between ampacity and forecasted ampacity. Negative (Positive) difference means that ampacity is overestimated (underestimated). A significant negative error can be critical for the TSO because this would lead to operate the system with a capacity higher than the actual limit of the line and threaten the security of the system. During $98 \%$ of the time, Ampacimon algorithm forecasted ampacities underestimates actual rating (secure forecast) with a maximal and acceptable error around $10 \%$ of the static limit.

The largest overestimated ampacities (maximal error up to $20 \%$ of static limit or 125 A here) happen, only $2 \%$ of time, which should be considered as of acceptable accuracy and very short compared to thermo-mechanical inertia to threaten the integrity of the system.

Forecasts underestimating the actual limits, although not desirable, are safe regarding the security of supply. However if capacities are systematically underestimated they might lead to a loss of opportunity like static limits do.

The accuracy of forecast models is critical and table 1 shows that quality of the models obtained is sufficient to be used as reliable information to operate the system closer to its actual limits. 


\section{Long-term forecast of network capacity}

Long-term forecasting is critical to plan system operation one or several days ahead. As long term forecast targets a period significantly longer than a few time constants of overhead line thermal behavior, it would seem obvious that weather forecast has to be used to predict accurately ampacity. However, in many practical cases, large discrepancies between ampacity predictions based on weather forecast and ampacity computed in real time have been observed. This can be highlighted for several consecutives hours [5] as shown in Figure 5. Maximal error can reach 50\% of actual rating.

Ongoing research aims to improve the forecast of conductor ampacity inferred from weather forecast only, this will be done in the coming future using an effective machine learning algorithm.

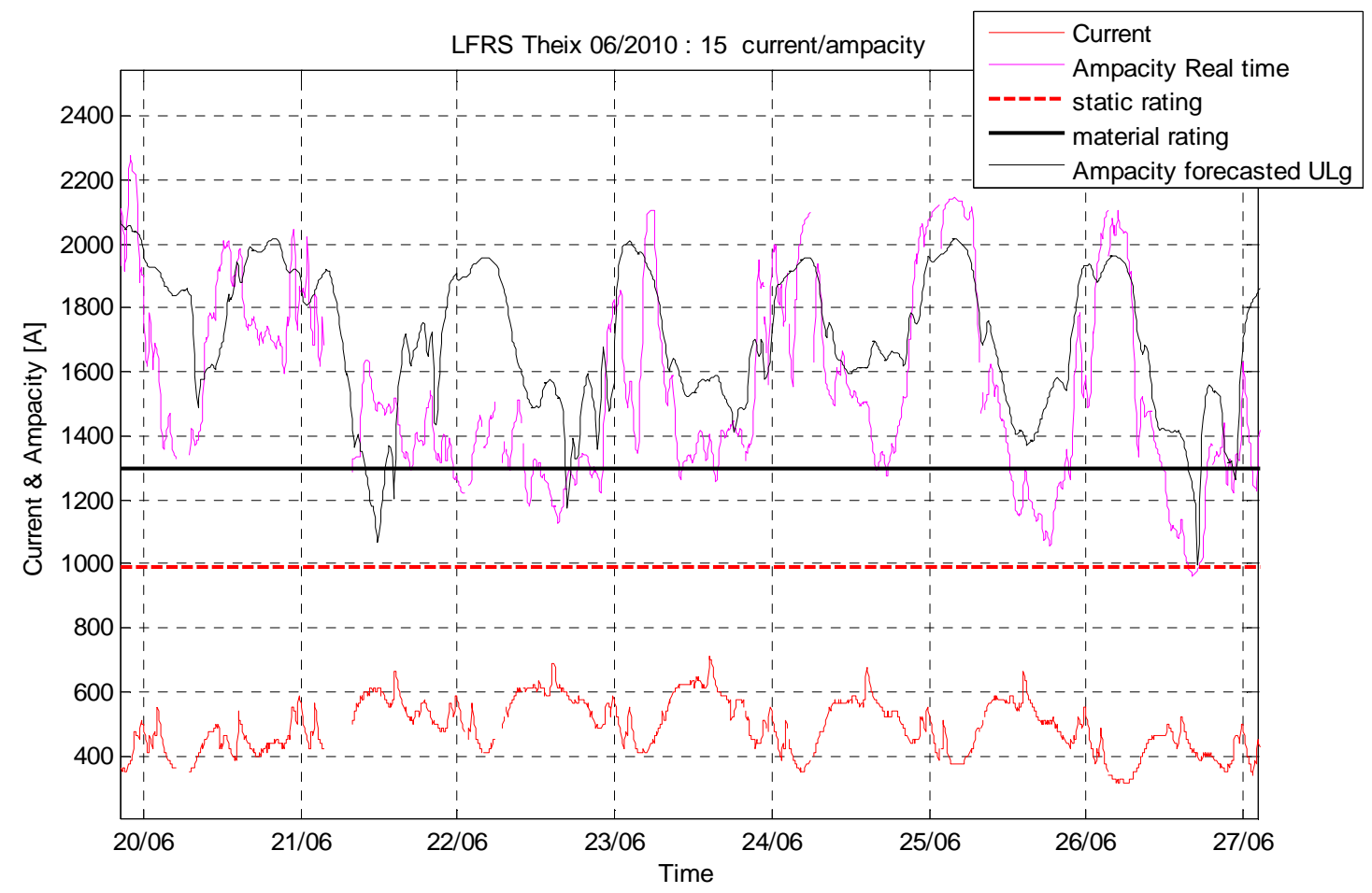

Figure 5 : Current, real time Ampacity, and forecasted ampacity (computed from weather forecast only).

It shall also be stressed that the final target is not to forecast weather variables, but to make a wise use of them to predict a highly reliable ampacity one-day ahead. This is the driving idea behind the machine learning.

In the past, up to today, as security of operation is a top priority in TSO's activities, it's common practice to operate the grid conservatively.

When using forecasting models, it is very important to know when to trust the prediction and when not to trust it. If it happens that the prediction has a high uncertainty TSO will chose to plan its operation in the most conservative way using static security limits. A forecasting model has to provide additional information regarding the reliability of its forecast. And this also can be done using data mining tools.

The aim must be to know (in advance!) when a forecast is reliable and can be trusted and when the situation is unpredictable and a conservative approach must be used.

\section{Future outlook and conclusions}

This paper has presented a smarter approach to predict the capacity of power networks using a combination of advanced network sensors and leading edge data-mining tools. Generalizing this approach to the network system as a whole is a challenging subject where more research is needed.

We have here presented a new approach focused on ampacity forecasting of power lines, using both local sensors and data mining tools. Until now we succeeded to forecast up to 4 hours ahead and we are deeply investigating a day ahead forecast, which is another challenge. Indeed weather forecasts are needed in 
such approach but they are clearly not enough to be used for a full reliable forecast of ampacity. Combining data mining and local observations during a significant amount of time on actual power lines behavior in their local environment where it is needed, we may approach what we need.

To couple dynamic line rating with a higher degree of flow supervision through wide-area network stability measurements and limits (Phase monitoring units, phase shifter device and WAMS) in our view represents the modernized grid that will be able to cope with tomorrow's requirements at an economically acceptable cost.

We have demonstrated that new sensor devices, such as Ampacimon, can really leverage big saving opportunities, if the flow of information they provide are carefully processed by modern data mining technologies. Not only these devices can provide a better insight of current situation, used together with other sources of information like weather forecast, operators will have a better visibility of short and long term behavior and properties of the transmission grid. This will definitely help operators to have more time and a better focus to work more in optimizing the use of the assets than in managing urgent corrective actions.

\section{References}

[1] JL Lilien, S. Guérard, B. Godard, J. Destiné. Microsystem array for live high voltage lines monitoring. CIGRE 2006, CIGRE Session papers, Group B2, B2-302.

[2] E. Cloet, JL. Lilien, P. Ferrières. Experiences of the Belgian and French TSOs using the "Ampacimon » real-time dynamic rating system. CIGRE 2010 Session papers, Group C2, C2-106

[3] E. Cloet, J.L. Lilien. Uprating Transmission Lines Through the Use of an Innovative Real-Time Monitoring System. IEEE Power \& Energy Society, ESMO conference. IEEE PES $12^{\text {th }}$ International Conference on Transmission and Distribution Construction, Operation and live-line maintenance, Providence, Rhodes Island, 16-19 May 2011.

[4] TWENTIES Project: UE Seventh Framework programme theme energy [Energy-2009-7 - Smart energy Networks], Annex I - "description of work", 2010, www.twenties-project.eu

[5] P. Schell, HM Nguyen, JL Lilien, Quantifying the limits of weather based dynamic line rating methods, CIGRE, Conference on Power Systems, Halifax, 2011

[6] www.ampacimon.com 